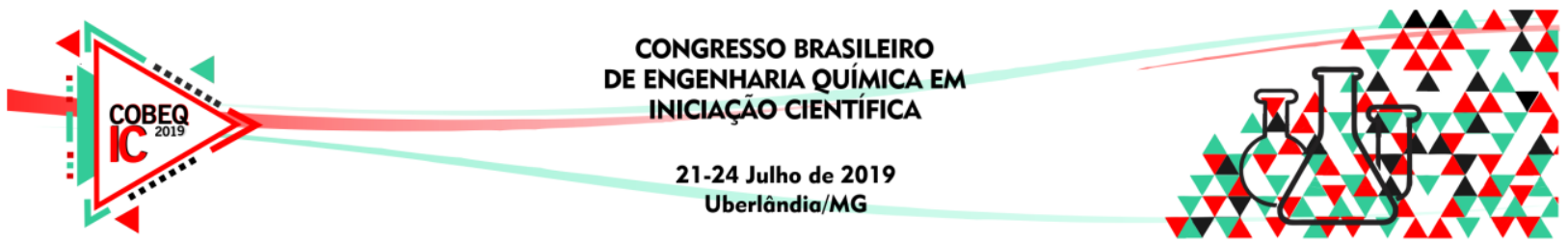

\title{
BIRREFRINGÊNCIA INDUZIDA POR ESCOAMENTO PARA OBTENÇÃO DO CAMPO DE TENSÕES EM POLÍMEROS FUNDIDOS
}

\author{
T.P.REIS ${ }^{1}$, A.R.SECCHI ${ }^{2}$, A.M.CASTRO ${ }^{2}$ e J.O.PEREIRA ${ }^{2}$ \\ ${ }^{1}$ Universidade Federal do Rio de Janeiro, Escola de Química \\ ${ }^{2}$ Universidade Federal do Rio de Janeiro, COPPE, Programa de Engenharia Química \\ E-mail para contato: thayna.preis@ hotmail.com
}

\begin{abstract}
RESUMO - Nas operações industriais com polímero fundido, o processamento é afetado diretamente por suas propriedades reológicas, sendo necessário conhecer o comportamento desses materiais nas condições práticas de escoamento para ser possível prever e avaliar a morfologia e as propriedades finais do material. Dessa forma, várias técnicas vêm sendo empregadas, com destaque para a birrefringência induzida pelo escoamento (Flow Induced Birefringence), observada durante o processamento de polímero fundido no reômetro multipasse capilar de duplo pistão (MultiPass Rheometer) acoplado a um módulo óptico. Neste trabalho, foi analisado o comportamento de um grade de polietileno linear de baixa densidade (LLDPE) fundido durante o escoamento através da geometria slit-die com 1,5 $\mathrm{mm}$ de comprimento do canal e $10,0 \mathrm{~mm}$ de profundidade no Reômetro Multipasse MPR5S em três temperaturas $\left(140^{\circ} \mathrm{C}, 150^{\circ} \mathrm{C}\right.$ e $\left.160^{\circ} \mathrm{C}\right)$ e três velocidades diferentes dos pistões: 0,1, 0,25 e 0,5 mm/s. Foi possível observar que com o aumento da temperatura houve uma redução na quantidade de franjas obtidas para a mesma velocidade de escoamento bem como uma redução nos valores de diferença de pressão. Em seguida, o software GIMP foi utilizado para a determinação do perfil do módulo da primeira diferença de tensões normais ao longo da linha central do escoamento para três velocidades de escoamento diferentes, mostrando que a metodologia via processamento das imagens de birrefringência é adequada e acurada para esse fim.
\end{abstract}

\section{INTRODUÇÃO}

O uso de produtos de origem polimérica vem se tornando uma necessidade cada vez mais constante na sociedade moderna, desde a produção de simples embalagens até a produção de materiais que requerem uma maior complexidade na sua utilização, como órgãos artificiais. Com esta finalidade, polímeros como polietileno, polipropileno, policarbonato e poliestireno são processados em elevadas temperaturas e são submetidos a altas taxas de deformação, gerando assim uma orientação e alongamento de suas cadeias, de forma que seu comportamento reológico deve ser bem compreendido. Os softwares desenvolvidos atualmente especializados em modelagem, simulação e otimização de processos e escoamento de polímeros não atendem às necessidades requeridas pelos processos produtivos. Desta forma, a possibilidade de utilizar a fluidodinâmica computacional na avaliação destes processos pode, além de outros benefícios, gerar uma redução significativa dos custos do 


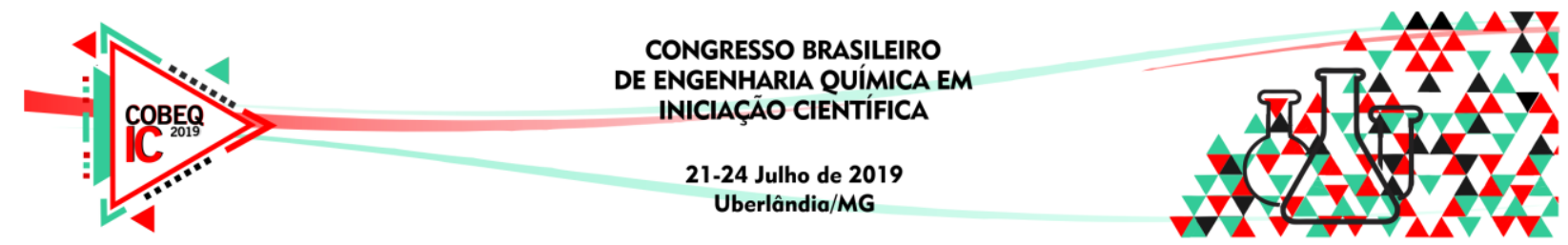

processo. Para uma compreensão científica do processamento de polímeros, o fluido fundido é submetido a um escoamento simples por cisalhamento sob condições de temperatura controlada, que pode ser realizada utilizando-se reômetros rotacionais ou extensionais.

Este trabalho tem por objetivo geral compreender o comportamento desses materiais durante condições reais de processamento. Assim, insere-se neste contexto a técnica de birrefringência induzida por escoamento (FIB: Flow Induced Birefringence), que possui grande potencial de aplicação na área de polímeros, porém ainda relativamente pouco explorada. Entre as principais características dessa técnica, pode-se citar: a quantidade observada ou medida é efeito da orientação molecular e permite a obtenção do perfil de tensões em toda a região de análise. O campo de tensão do escoamento é quantificado em termos da diferença de tensão principal (PSD: Principal Stress Diference), que na linha central do escoamento pode ser escrita conforme mostrado na Equação (1).

$$
|P S D|=\left|\tau_{11}-\tau_{22}\right|=\left|N_{1}\right|=\frac{k \lambda}{|C| d}
$$

Sendo $\left|\mathrm{N}_{1}\right|$ o módulo da primeira diferença de tensões normais, $k=0,1,2, \ldots$, a ordem da franja, $\lambda$ o comprimento de onda da luz polarizada que incide sobre o meio birrefringente, $C o$ coeficiente de tensão óptico que estabelece a relação entre o tensor tensão e o tensor índice de refração e $d$ o comprimento do meio anisotrópico (maiores informações em COLLINS e MACKLEY, 2005; FARIAS et al., 2014; LEE e MACKLEY, 2001).

Neste trabalho são realizadas a obtenção e análise de imagens de birrefringência induzida por escoamento no MPR5S de uma amostra de polietileno linear de baixa densidade, de forma que seja possível avaliar o comportamento reológico do material, bem como, o estudo e desenvolvimento de modelos constitutivos para a simulação de escoamento de fluidos complexos e a validação de modelos fenomenológicos para a simulação do escoamento de fluidos viscoelásticos.

\section{METODOLOGIA}

As imagens de birrefringência obtidas neste trabalho foram geradas através de experimentos realizados no Reômetro Multipasse (MPR: MultiPass Rheometer). O MPR (Figuras 1.a, 1.b, 1.c) é um reômetro capilar de duplo pistão, desenvolvido por Mackley e colaboradores, (MACKLEY e HASSEL, 2011), University of Cambridge (UK). A etapa de carregamento da amostra no MPR foi realizada conforme descrito em Farias et al. (2014). A tensão é aplicada na amostra através da movimentação dos pistões, axialmente opostos, contra a amostra, forçando seu movimento pela seção de teste, localizada na região central do equipamento. Em seguida, a birrefringência induzida é capturada por uma câmera de alta resolução e as imagens obtidas na região de diferença de pressão constante são tratadas no software GIMP (GNU Image Manipulation Program). 


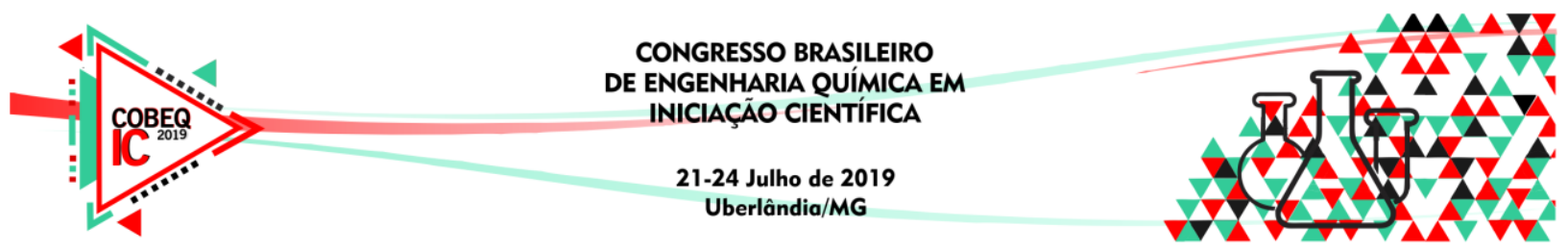

Figura 1 - Reômetro Multipasse

a) Visão geral do equipamento

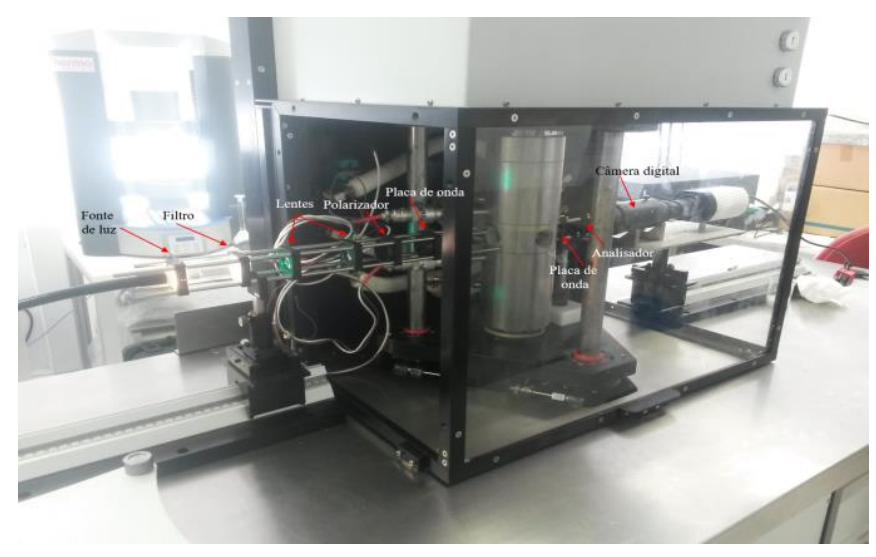

b) Geometria slit-die

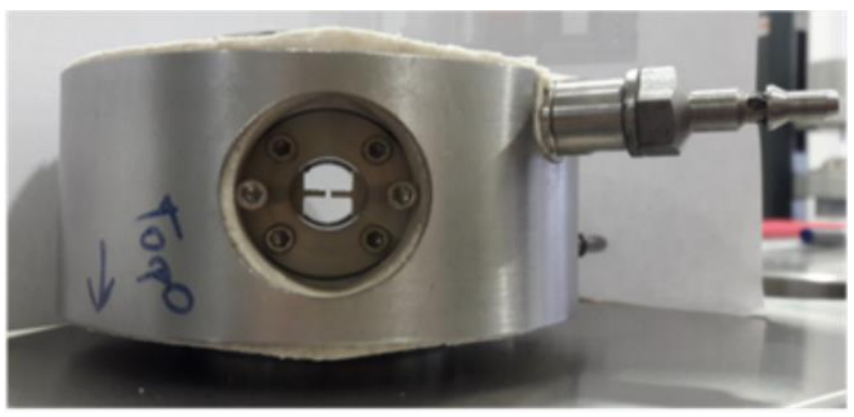

c) Montagem dos barris do equipamento

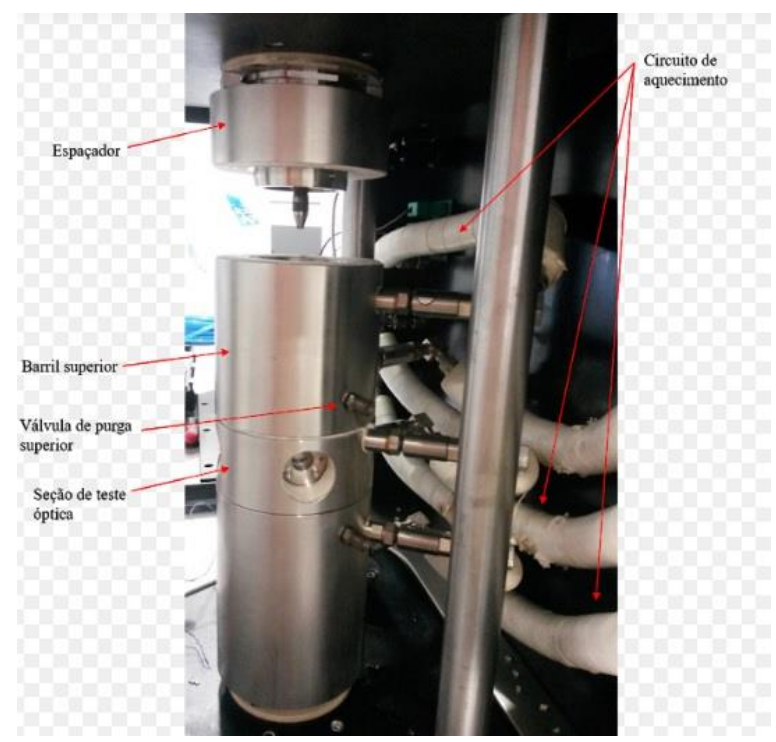

O fato de o escoamento ter sido inteiramente filmado com uma câmera de alta resolução, garantiu assim a captação das imagens de birrefringência. As imagens obtidas durante o período de diferença de pressão constante foram posteriormente processadas com o software GIMP (GNU Image Manipulation Program), que permite a medição da distância dos centros das franjas escuras ao longo da linha central do escoamento e, consequentemente, a obtenção do campo de tensões e a determinação da primeira diferença de tensão normal na linha central do escoamento. O período de diferença de pressão constante foi utilizado para o processamento das imagens pois ele garante que o escoamento esteja desenvolvido e que os padrões de franjas brilhantes e escuras estejam totalmente estabelecidos.

As temperaturas utilizadas no experimento foram: $140^{\circ} \mathrm{C}, 150^{\circ} \mathrm{C}$ e $160^{\circ} \mathrm{C}$; a direção do escoamento através da geometria slit-die foi de baixo para cima em diferentes velocidades dos pistões $(0,1,0,25$ e $0,5 \mathrm{~mm} / \mathrm{s})$; para a obtenção dos resultados numéricos foi considerado 


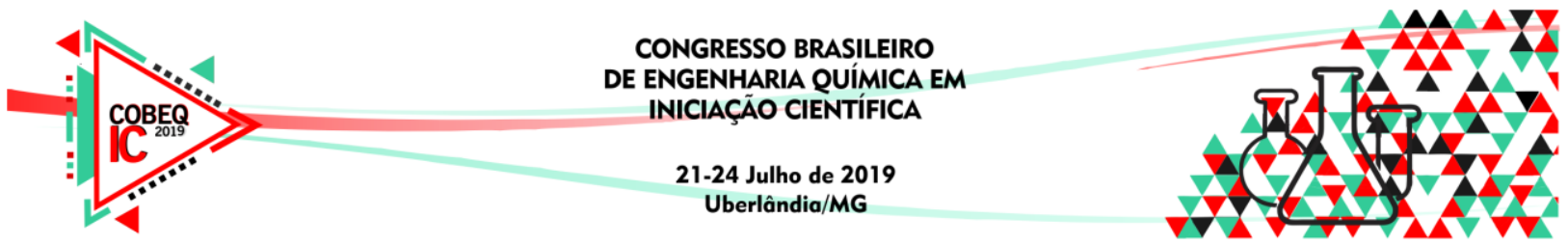

$C=2,2 \times 10^{-9} \mathrm{~Pa}^{-1}$ para o polietileno e utilizada luz polarizada monocromática de comprimento de onda de $514 \mathrm{~nm}$.

\section{RESULTADOS}

A Figura 2 mostra o comportamento da queda de pressão na slit-die em função do tempo para os experimentos realizados na temperatura intermediária de $150^{\circ} \mathrm{C}$. Observa-se que os experimentos de menor velocidade apresentam períodos de estado estacionário mais longos, permitindo assim a obtenção de um maior número de imagens em estado estacionário. Apesar de apresentar um período de diferença de pressão constante menor, a alta capacidade de captura de quadros da câmera utilizada permitiram imagens de boa qualidade, garantindo assim uma análise satisfatória das imagens.

Figura 2 - Queda de pressão a $150^{\circ} \mathrm{C}$

Figura 3 - Queda de pressão a $0,25 \mathrm{~mm} / \mathrm{s}$
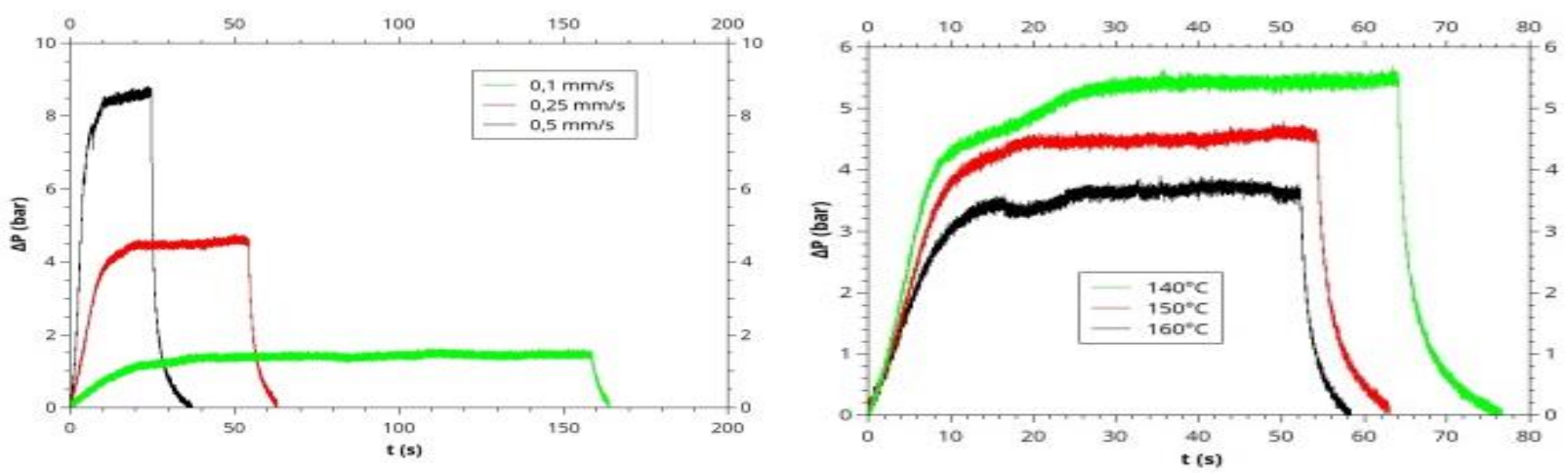

A Figura 3 mostra a primeira diferença de tensão normal ao longo da linha central do escoamento na velocidade intermediária de $0,25 \mathrm{~mm} / \mathrm{s}$. Observa-se que, com o aumento da temperatura, há uma redução nos valores de diferença de pressão ao longo da linha central do escoamento. Isso ocorre pois o aumento da temperatura proporciona a redução da viscosidade do fundido.

Algumas das imagens obtidas durante o período de diferenças de pressão constante são exibidas nas Figuras 4, 5 e 6 nas suas respectivas velocidades. Elas foram utilizadas para a contagem dos padrões de franjas no eixo central do escoamento. A numeração das franjas $(k)$ foi baseada no trabalho de Scelsi (2009). É possível visualizar as zonas de maiores e de maiores tensões como áreas escuras localizadas, respectivamente, nos lados das inserções da fenda e na entrada na fenda nas Figuras 4, 5 e 6.

Como esperado, a queda de pressão total através da slit-die e o número observado de franjas aumentou com o aumento da velocidade dos pistões e nenhuma instabilidade ou comportamento anormal foi encontrado. Em cada experimento um total de 15 imagens foram analisadas usando a metodologia proposta por Castro et al. (2017).

Figura 4 - Numeração das franjas (k) a $140^{\circ} \mathrm{C}$. Em (a) 0,1 ; (b) 0,25 ; (c) $0,5 \mathrm{~mm} / \mathrm{s}$.

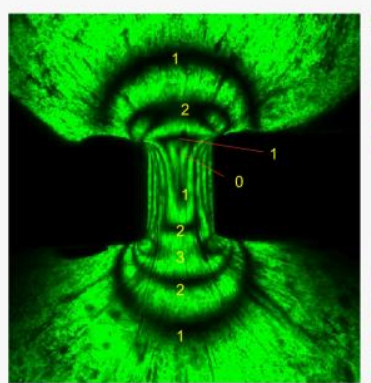

(a)

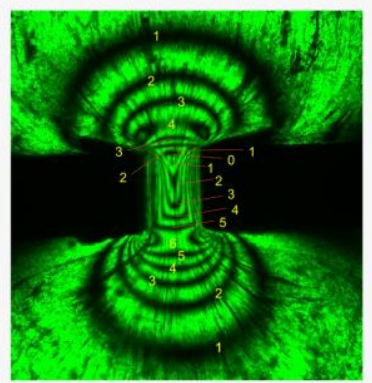

(b)

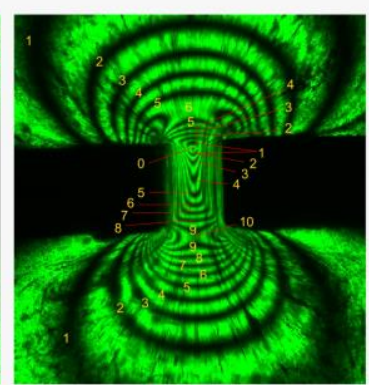

(c) 


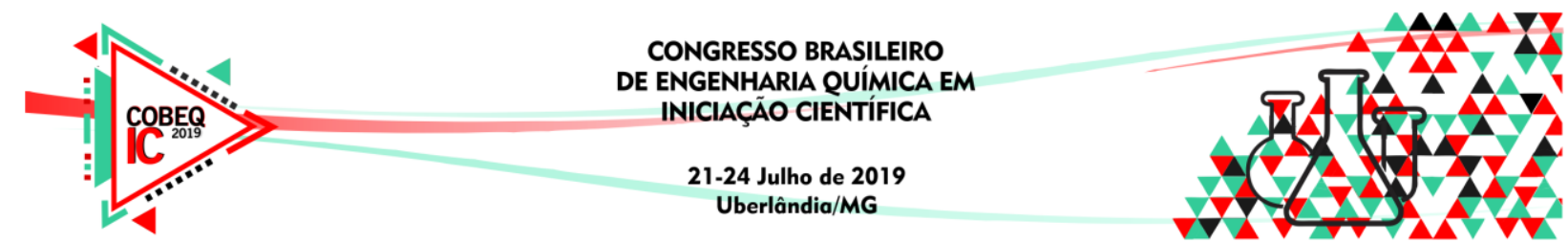

Figura 5 - Numeração das franjas (k) a $150^{\circ} \mathrm{C}$. Em (a) 0,1 ; (b) 0,25 ; (c) $0,5 \mathrm{~mm} / \mathrm{s}$.

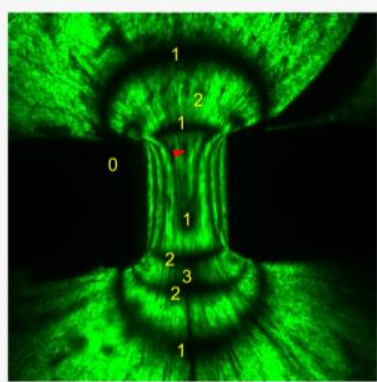

(a)

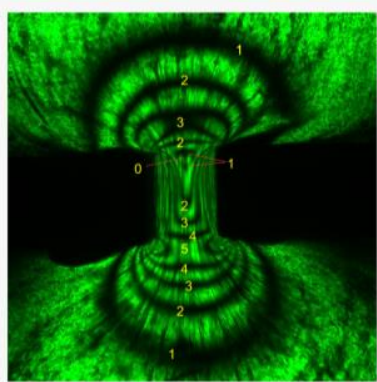

(b)

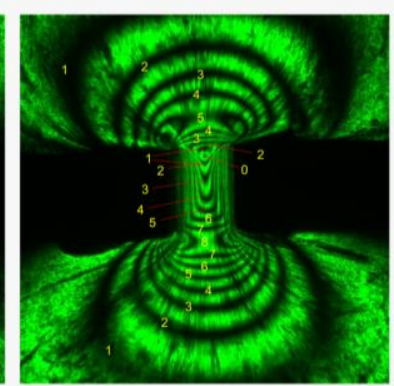

(c)

Figura 6 - Numeração das franjas (k) a $160^{\circ} \mathrm{C}$. Em (a) 0,1 ; (b) 0,25 ; (c) $0,5 \mathrm{~mm} / \mathrm{s}$.

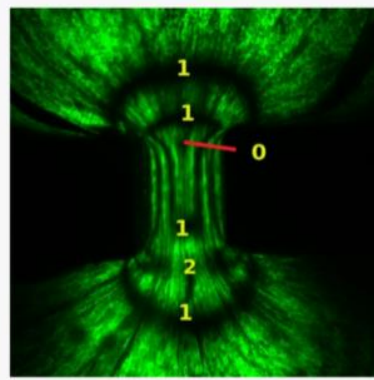

(a)

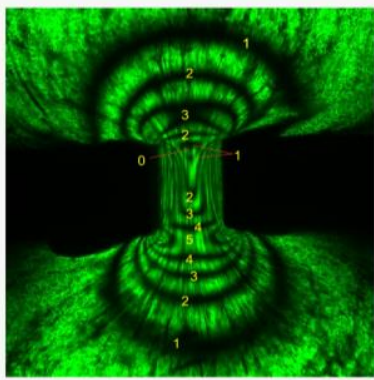

(b)

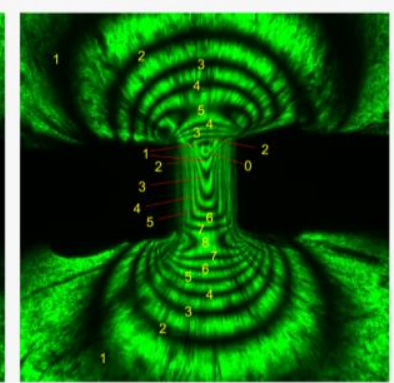

(c)

A numeração das franjas $(k)$ foi realizada de acordo com o seguinte raciocínio: $k=0$ longe da região de entrada e atinge um ponto máximo na entrada da fenda ( $k=3$ na Figura $4 \mathrm{a}, k=5$ na Figura $4 \mathrm{~b}, k=10$ na Figura $4 \mathrm{c}$ ).

O módulo da primeira diferença de tensões normais $\left(\left|N_{1}\right|\right)$ foi obtido partindo-se da Equação 1 e da contagem das franjas escuras a partir do software GIMP. Esta quantificação resultou no gráfico visualizado na Figura 7, onde é mostrada a posição $x=0$ como a região de entrada da fenda. Percebe-se um comportamento padrão para as três velocidades testadas, onde na região de entrada da fenda predomina a resposta não linear do material, visto que $\left|\mathrm{N}_{1}\right|$ aumenta de forma acentuada nesta região até o ponto de cela, observado nas Figuras 4, 5 e 6. Após este ponto, as tensões relaxam ao longo da linha central. A região escura na saída da fenda, observada como um círculo preto nas imagens das Figuras 4, 5 e 6, é a região onde há a troca da direção principal do escoamento de forma que $\tau_{y y}$ e $\tau_{x x}$ se tornam iguais e o $\left|N_{1}\right|$ é igual a zero.

Figura 7 - $\left|\mathrm{N}_{1}\right|$ em função da linha central do escoamento para as três velocidades estudadas

(a) sob as três velocidades

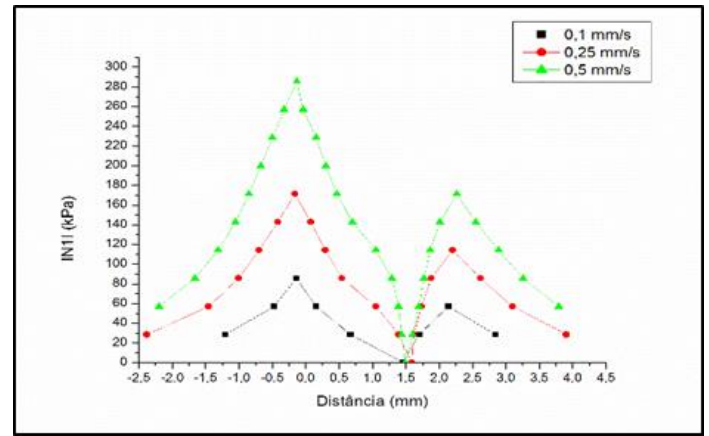

(b) sob as três temperaturas de teste.

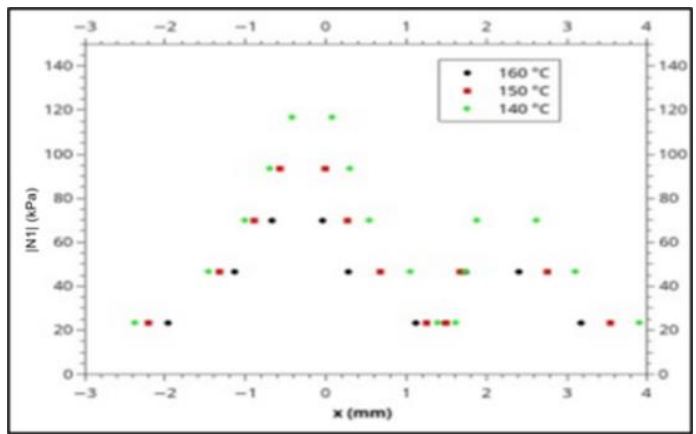




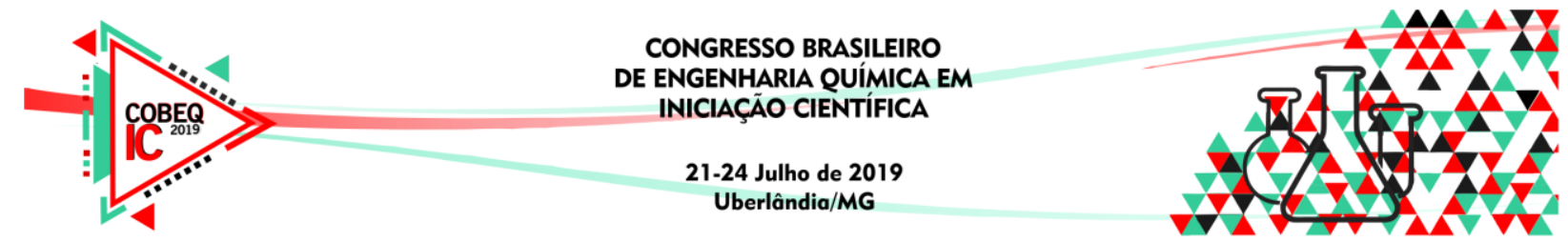

\section{CONCLUSÃO}

De acordo com os resultados obtidos pelo experimento, foi possível verificar a dependência não linear do comportamento do padrão das franjas com o aumento da velocidade do pistão. Adicionalmente, foi comprovado que a metodologia para a determinação da posição dos centros das franjas escuras ao longo da linha central do escoamento a partir do software GIMP é adequada e apresentou resultados coerentes com o esperado, permitindo assim a obtenção do perfil do módulo da primeira diferença de tensões normais ao longo da linha central do escoamento. Finalmente, pode-se verificar que os experimentos reo-ópticos possuem grande potencial para a compreensão das propriedades viscoelásticas de fluidos poliméricos em condições reais de processamento, o que é importante para a melhoria de equipamentos industriais e otimização de processos industriais.

\section{REFERÊNCIAS}

CASTRO, A.M., PEREIRA, J.O.; FARIAS, T.M.; SECCHI, A.R.; CARDOZO, N.S.M., "Application of the GIMP software in the analysis of birefringence images obtained in a multipass rheometer", Rheol. Acta, v.57, p.113 - 126, 2017.

COLLIS, M, MACKLEY, M. The melt processing of monodisperse and polydisperse polystyrene melts within a slit entry and exit flow, J. Non-Newtonian Fluid Mech. v. 128, p. 29-41, 2005.

FARIAS, T.M., BUTLER, S., SECCHI, A. R., CARDOZO, N.S.M. "Utilização da técnica de birrefringência em reômetro multipasse para a diferenciação de grades de poliestireno cristal", Polímeros, v. 24, n.5, p. 596-603, 2014.

LEE, K., MACKLEY, M.R., "The application of the multi-pass rheometer for precise rheooptic characterisation of polyethylene melts", Chem. Eng. Sci., v. 56, n. 19, p. 56535661, 2001.

MACKLEY, M.R.; HASSELL, D.G. The multipass rheometer a review, J. Non-Newtonian Fluid Mech., v. 166, n. 9-10, p. 421-456, 2011. 